\title{
Finite Element Analysis on Nanomechanical Sensing of Cellular Forces
}

\author{
Gaku Imamura, ${ }^{*}, * * \dagger$ Kota ShIBA,* and Genki YoshIKAWA $* * * *$ \\ *World Premier International Research Center Initiative (WPI), International Center for Materials \\ Nanoarchitectonics (MANA), National Institute for Materials Science (NIMS), Tsukuba, Ibaraki 305-0044, \\ Japan \\ **International Center for Young Scientists (ICYS), National Institute for Materials Science (NIMS), Tsukuba, \\ Ibaraki 305-0044, Japan \\ ***Materials Science and Engineering, Graduate School of Pure and Applied Science, University of Tsukuba, \\ 1-1-1 Tennodai, Tsukuba, Ibaraki 305-8571, Japan
}

\begin{abstract}
Detection of cellular forces plays an important role in investigating the mechanical basis of cells. As nanomechanical sensors can directly detect surface stress, they can be utilized to detect cellular forces. In the present study, we perform quantitative simulations of nanomechanical sensors for the detection of cellular forces using finite element analyses (FEA). We focus on two types of nanomechanical sensors: a cantilever-type sensor and a membrane-type surface stress sensor (MSS). It is found that sensing signals can be obtained when cells on the nanomechanical sensors synchronize their motions. To effectively detect cellular forces on the nanomechanical sensors, we discuss the optimization scheme for a coating layer on the surface of the sensors.
\end{abstract}

Keywords Cell motility, mechanobiology, nanomechanical sensors, cantilever-type sensors, membrane-type surface stress sensors (MSS), finite element analysis (FEA)

(Received July 9, 2016; Accepted September 8, 2016; Published November 10, 2016)

\section{Introduction}

Nanomechanical sensors have been emerging as a new type of sensor that can be applied to various fields. Nanomechanical sensors basically detect the two basic physical parameters: volume and/or mass. As all substances have volume and mass, almost any kind of substance should be detectable with the use of nanomechanical sensors. Many studies using nanomechanical sensors have been reported so far, ranging from gases to biological molecules. ${ }^{1,2}$ Not only molecules but also microorganisms such as viruses and bacteria can be detected by nanomechanical sensors. ${ }^{3,4}$ Since the first reports on nanomechanical sensing in 1994, 5,6 cantilever-type sensors have been widely used as a typical nanomechanical sensor. In 2011, a membrane-type surface stress sensor (MSS) appeared as a practical sensing platform. ${ }^{7}$

While mechanobiology has been attracting much attention as a new field of cell biology, ${ }^{8}$ application of nanomechanical sensors to mechanobiology is one of the most interesting and challenging issues. In mechanobiology, it is important to evaluate the mechanical forces caused by cells in order to investigate how cells sense physical forces and respond to them. To measure the forces exerted through cellular functions such as adhesion, migration, and proliferation, micropatterned substrates

$\dagger$ To whom correspondence should be addressed.

E-mail: IMAMURA.Gaku@nims.go.jp including micropillar substrates are frequently used. ${ }^{9,10}$ However, it is known that the substrate topography causes a certain effect on the cellular functions. ${ }^{11,12}$ Nanomechanical sensors, on the other hand, can reduce such problems and might provide a new sensing platform with a plane surface.

In this study, we investigate the nanomechanical sensing of cellular forces by means of finite element analysis (FEA) and evaluate the feasibility of nanomechanical sensors for mechanobiology. We performed FEA simulations on two kinds of nanomechanical sensors, a cantilever-type sensor and an MSS. The effects of cell position, cell size, the number of cells, and phase (spreading and contraction) are comprehensively investigated. We also investigate the optimization scheme for coating the sensors to amplify the sensing signals.

\section{Materials and Methods}

FEA simulations were performed in COMSOL Multiphysics $5.1^{\circledR}$ with the Structural Mechanics module. Figure 1 depicts the principle of nanomechanical sensing of cellular forces. In Fig. 1, a spreading cell applies a surface stress on the sensors, leading to the deformation of the substrate or the membrane. Note that, in the case of FEA, this behavior is simulated by setting an initial compressive stress which induces resultant tensile stress when it is equilibrated. The resultant deflection $d$ can be detected as a sensing signal for a cantilever-type sensor. In the case of an MSS, the surface stress on the membrane is 
Cantilever-type sensor

(a)
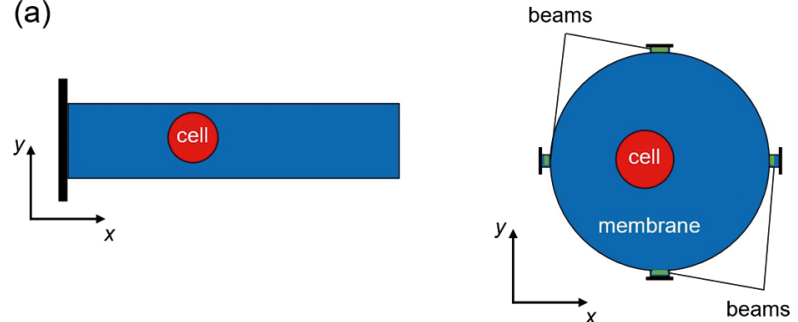

(b)
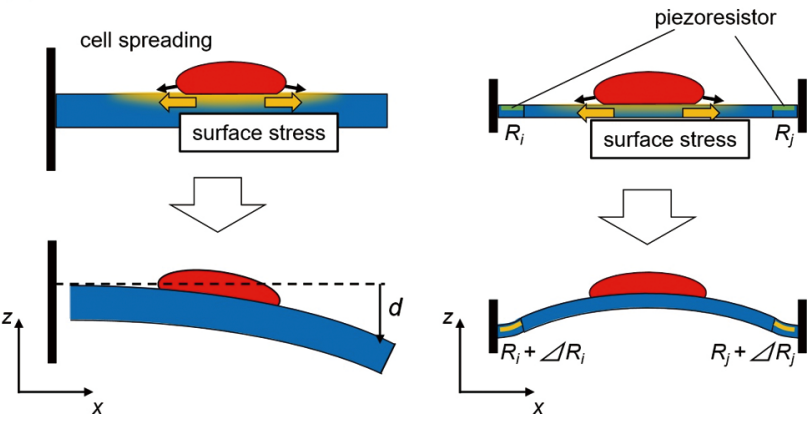

Fig. 1 Schematic illustrations of two-types of nanomechanical sensors: a cantilever-type sensor and an MSS. (a) Top view and (b) side view of the sensors.

transduced to the four beams as an amplified uniaxial stress, resulting in the changes in electrical resistance of the piezoresistors embedded in the beams. In the present simulation, a cell was modeled as a circle with a radius of $r \mu \mathrm{m}$ applying a constant surface stress on a nanomechanical sensor. As Bischofs et al. reported that the surface stress caused by an endothelial cell was $2 \times 10^{-3} \mathrm{~N} / \mathrm{m}$, the surface stress for each cell was set at $2 \times 10^{-3} \mathrm{~N} / \mathrm{m}$ (applying $2 \times 10^{5} \mathrm{~N} / \mathrm{m}^{2}$ bulk stress on a $10 \mathrm{~nm}-$ thick film $\left(2 \times 10^{5}\left[\mathrm{~N} / \mathrm{m}^{2}\right] \times 10 \times 10^{-9}[\mathrm{~m}]=2 \times 10^{-3}[\mathrm{~N} / \mathrm{m}]\right)$, simulating the surface stress component of a cell). ${ }^{13}$ Positive and negative surface stresses correspond to tensile (cell spreading) and compressive stress (cell contraction), respectively.

\section{Results}

Firstly, we performed FEA simulations on a cantilever-type sensor. The dimension of the cantilever was set at a typical geometry for sensing applications $(500 \times 100 \times 1 \mu \mathrm{m})$ as shown in Fig. 2a. Figure $2 b$ shows the dependence of the deflection of the cantilever $d$ on the position of a cell. A cell was located $x$ $\mu \mathrm{m}$ from the fixed-end. The radius of the cell $r$ was fixed at $10 \mu \mathrm{m}$. Tensile stress is applied in the simulation, while compressive stress results in negative $d$ with the same absolute value. As expected from basic mechanics, $d$ becomes larger as the cell gets closer to the fixed-end. The dependence of $d$ on $r$ is shown in Fig. 2c. When $x$ is fixed at $50 \mu \mathrm{m}, d$ is proportional to the square of $r$, suggesting that $d$ is proportional to the area covered with the cell. This linear dependence on the area can be also seen in Fig. 3. We investigated the dependence of $d$ on the number of cells. Figure $3 \mathrm{a}$ depicts the configuration of the simulated system where multiple cells exist. In the simulation, the center of the multiple cells was set at $50 \mu \mathrm{m}$ from the fixedend. As shown in Fig. 3b, $d$ is proportional to the number of cells. The data points in Figs. $2 \mathrm{c}$ and $3 \mathrm{~b}$ are plotted in Fig. $3 \mathrm{c}$. (a)
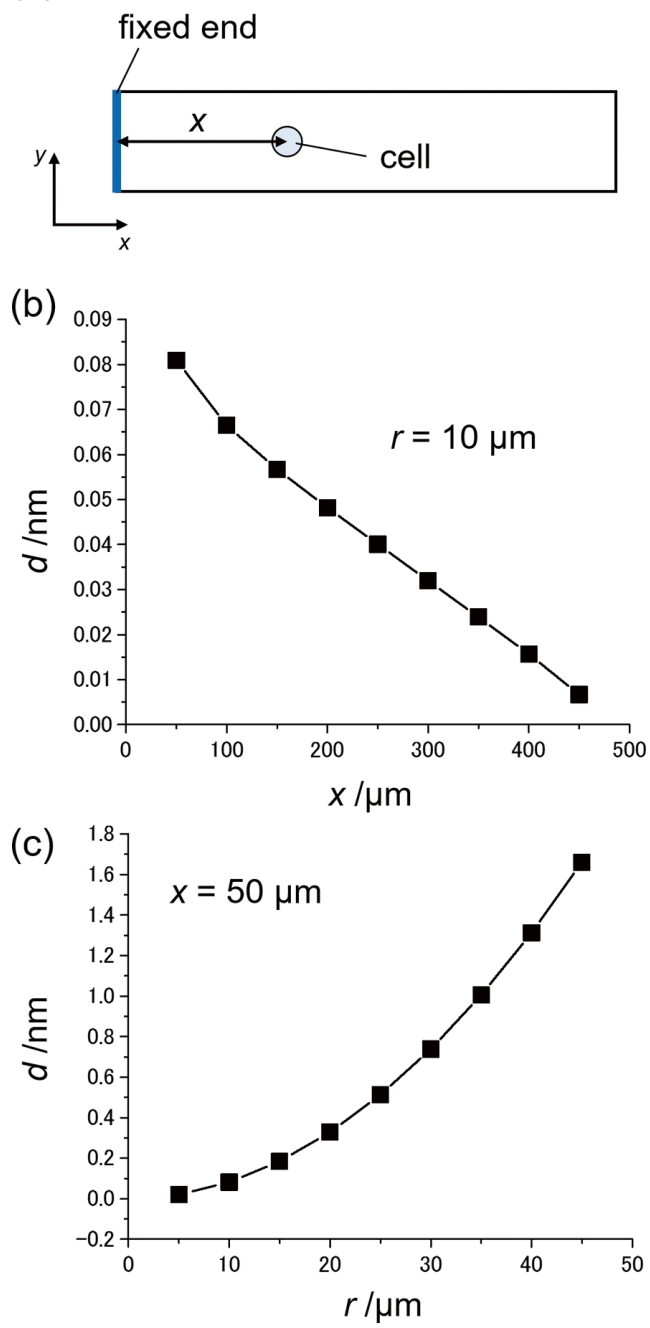

Fig. 2 (a) Schematic illustration of a cantilever-type sensor and a cell. (b) Dependence of the deflection $d$ on the cell position $x$ ( $r=$ $10 \mu \mathrm{m})$. (c) Dependence of the deflection $d$ on the radius of the cell $r$ $(x=50 \mu \mathrm{m})$.

It is obvious that $d$ increases linearly with the area covered with cells as long as the center position of the cells is regarded as constant.

To simulate a multiple-cell system, it is also important to consider the phase of cells; positive (tensile) and negative (compressive) stresses are induced through cell spreading and contraction, respectively. The absolute values for the positive and negative stresses are set to be the same. We performed FEA simulation for a two-cell system where the phase of the cells is taken into account. The configuration and the phases of the cells are shown in Fig. 4. The radii of the cells were set at $10 \mu \mathrm{m}$. The red and blue cells represent spreading and contracting cells, respectively. Two cells were set at the positions $50 \mu \mathrm{m}$ from the fixed-end. Two types of alignments (i.e. $x$-aligned and $y$-aligned) were simulated for comparison. As shown in Fig. 4, large $d$ can be obtained for in-phase cells, while zero or very small $d$ can be found for out-of-phase cells. Although $x$-aligned cells cause non-zero $d$ even for out-of-phase owing to the asymmetric configuration (red circles in Fig. 3), $d$ is much smaller than that for the in-phase cells.

Secondly, we performed the simulations on an MSS. 
(a) $N=2$

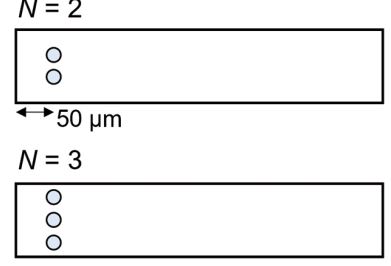

\begin{tabular}{|c|}
\hline $\begin{array}{l}1+-0 \\
00 \\
00 \\
00\end{array}$ \\
\hline$\longleftrightarrow 50 \mu \mathrm{m}$ \\
\hline$N=9$ \\
\hline $\begin{array}{l}000 \\
000 \\
000 \\
000\end{array}$ \\
\hline
\end{tabular}

(b)

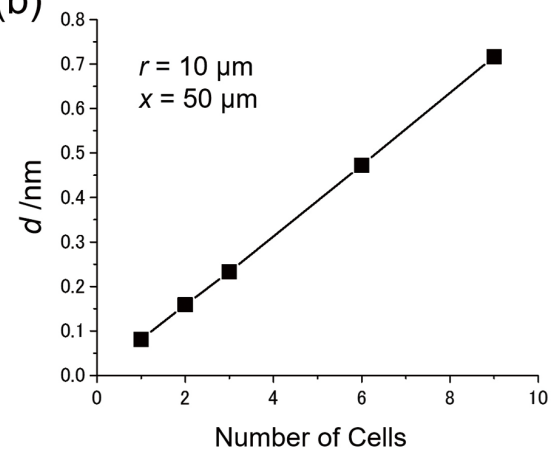

(c)

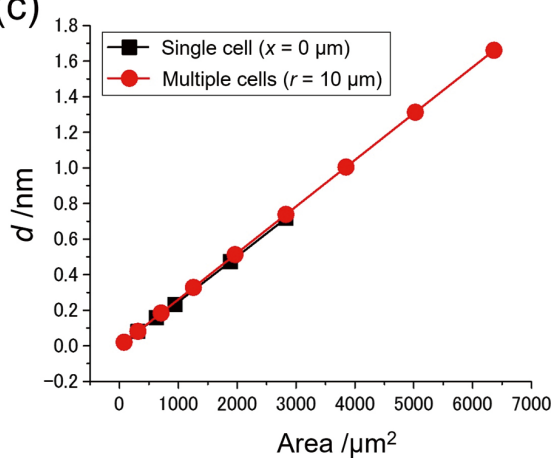

Fig. 3 (a) Configuration of multiple cells on the cantilever-type sensor. (b) Dependence of the deflection $d$ on the number of cells $(r=10 \mu \mathrm{m}, x=50 \mu \mathrm{m})$. (c) Dependence of the deflection $d$ as a function of the area covered by cells. Black squares and red circles are from the data points shown in Figs. $3 \mathrm{~b}$ and 2c, respectively.

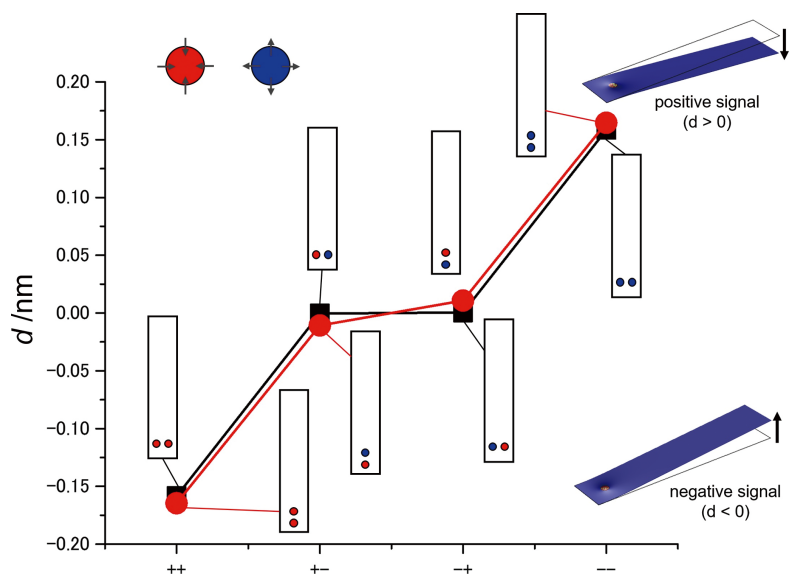

Fig. 4 Dependence of the deflection $d$ on the phase difference between the two cells. Red and blue cells represent positive (tensile) and negative (compressive) stresses, respectively.

The dimensions for the MSS were set according to the previous report. ${ }^{14}$ The diameter and the thickness of the membrane were 500 and $2.5 \mu \mathrm{m}$, respectively (Fig. 5). The membrane is suspended by the four beams, in which piezoresistors are embedded $\left(R_{1}-R_{4}\right)$. The dimensions of each beam in direction $x \times y$ are as follows: sensing beams for $R_{1}$ and $R_{3}, 10 \times 16 \mu \mathrm{m}$; sensing beams for $R_{2}$ and $R_{4}, 26 \times 10 \mu \mathrm{m}$. The total output signal $V_{\text {out }}$ obtained from the Wheatstone bridge circuit composed of the four piezoresistors can be approximately given by the following equation:

$$
V_{\text {out }}=\frac{V_{\mathrm{B}}}{4}\left(\frac{\Delta R_{1}}{R_{1}}-\frac{\Delta R_{2}}{R_{2}}+\frac{\Delta R_{3}}{R_{3}}-\frac{\Delta R_{4}}{R_{4}}\right)=\frac{V_{\mathrm{B}}}{4}\left(\frac{\Delta R_{\mathrm{tot}}}{R_{\mathrm{tot}}}\right),
$$

where $V_{\mathrm{B}}$ is the bridge voltage applied to the circuit and $\Delta R_{\mathrm{i}} / R_{\mathrm{i}}$ is the relative resistance change in $R_{\mathrm{i}}{ }^{7} R_{\mathrm{tot}}$ is the total resistance (a)

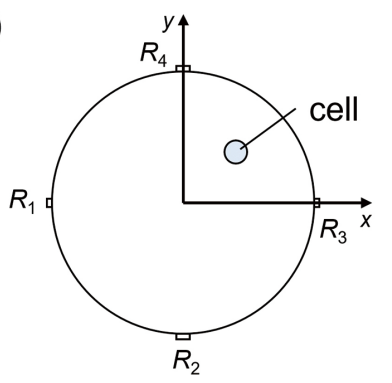

(b)
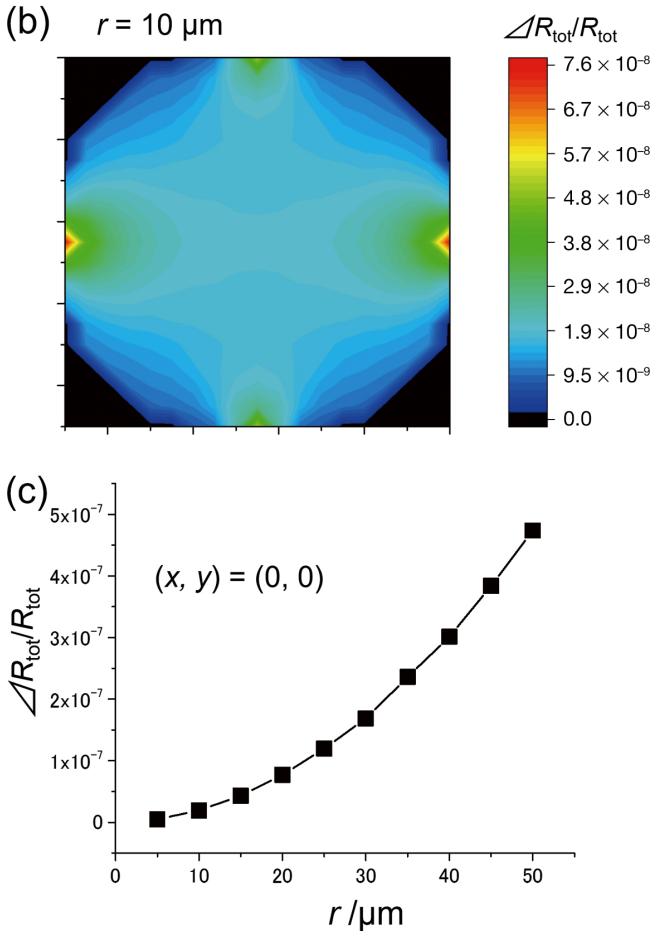

Fig. 5 (a) Schematic illustration of an MSS and a cell. (b) Dependence of $\Delta R_{\mathrm{tot}} / R_{\mathrm{tot}}$ on the cell position $(x, y)(r=10 \mu \mathrm{m})$. (c) Dependence of $\Delta R_{\mathrm{tot}} / R_{\mathrm{tot}}$ on the radius of the cell $r(x=0 \mu \mathrm{m}, y=0 \mu \mathrm{m})$. 
(a)

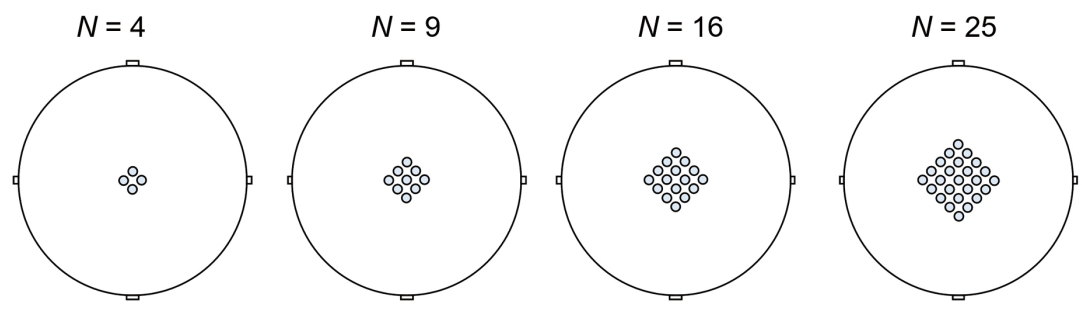

(b)

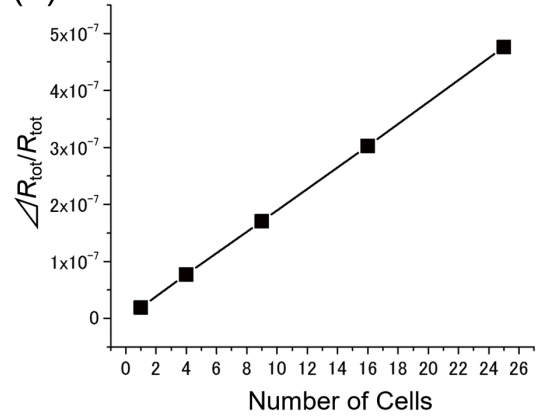

(C) Single cell $(x=0 \mu \mathrm{m}, y=0 \mu \mathrm{m})$

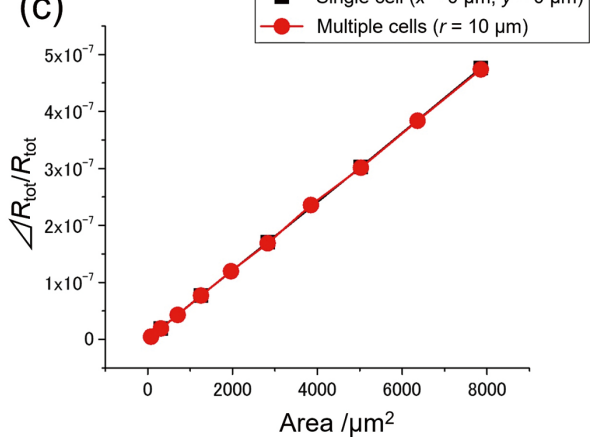

Fig. 6 (a) Configuration of multiple cells on the MSS. (b) Dependence of $\Delta R_{\mathrm{tot}} / R_{\mathrm{tot}}$ on the number of cells $(r=10 \mu \mathrm{m})$. (c) Dependence of $\Delta R_{\mathrm{tot}} / R_{\mathrm{tot}}$ as a function of the area covered by cells. Black squares and red circles are from the data points shown in Figs. $6 \mathrm{~b}$ and $5 \mathrm{c}$, respectively.

of the Wheatstone bridge circuit. Figure $5 \mathrm{~b}$ shows the dependence of $\Delta R_{\text {tot }} / R_{\text {tot }}$ on the position of a cell plotted as a color gradient. Similar to a cantilever-type sensor, a cell near a fixed-end (a beam) tends to give a higher sensing signal. In the case of MSS, however, a certain position close to one beam is far from other beams at the same time, resulting in rather complicated dependence on the cell position. Figure $5 \mathrm{c}$ shows the relationship between $\Delta R_{\mathrm{tot}} / R_{\mathrm{tot}}$ and $r$, resulting in a parabola. FEA simulations were also performed for multi-cell systems as shown in Fig. 6a. The cells were placed so that the center of the cells was set at the center of the membrane. Each cell applies a positive (tensile) surface stress on the membrane. $\Delta R_{\mathrm{tot}} / R_{\mathrm{tot}}$ increases linearly with the number of cells. The data points shown in Figs. $5 \mathrm{c}$ and $6 \mathrm{~b}$ fall onto the same line when they are plotted as a function of the area covered with cells (Fig. 6c), indicating that $\Delta R_{\mathrm{tot}} / R_{\mathrm{tot}}$ is proportional to the covered area.

Two-cell systems considering the phase of cells were also investigated for the MSS. Compared to single-cell systems, larger $\Delta R_{\mathrm{tot}} / R_{\mathrm{tot}}$ can be obtained for in-phase cells. If the two cells are located near the center of the membrane, $\Delta R_{\mathrm{tot}} / R_{\mathrm{tot}}$ becomes zero for the out-of-phase cells because of the symmetric configuration (Fig. 7a). In the case that the two cells are near a beam $\left(R_{3}\right)$, non-zero $\Delta R_{\text {tot }} / R_{\text {tot }}$ can be obtained for $x$-aligned cells even when they are out-of-phase (Fig. 7b). However, $\Delta R_{\mathrm{tot}} / R_{\mathrm{tot}}$ for the out-of-phase cells is approximately three times smaller than that for the in-phase cells.

On the basis of the FEA simulations for the cantilever-type sensor and the MSS, it seems important for the detection of cellular forces that cells on a nanomechanical sensor synchronize their motions. We performed FEA simulations on multiple-cell systems where the surfaces of the sensors are covered with cells (Fig. 8). If all the cells on the sensor are in-phase, enhanced sensing signals can be obtained both for the cantilever and the MSS. In contrast, no detectable signals are expected for the multiple-cell systems where the cells are not in-phase. It should
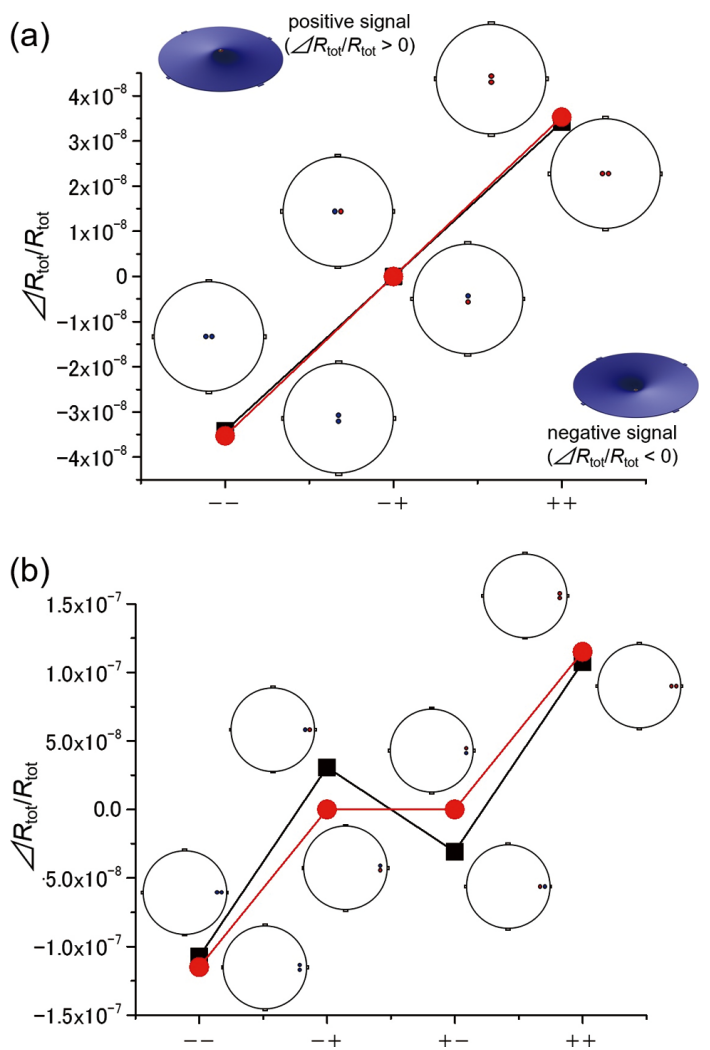

Fig. 7 Dependence of $\Delta R_{\mathrm{to}} / R_{\mathrm{tot}}$ on the phase difference between the two cells. (a) Two cells are around the center of the membrane. (b) Two cells are near $R_{3}$. Red and blue cells represent positive (tensile) and negative (compressive) stresses, respectively. 
(a)

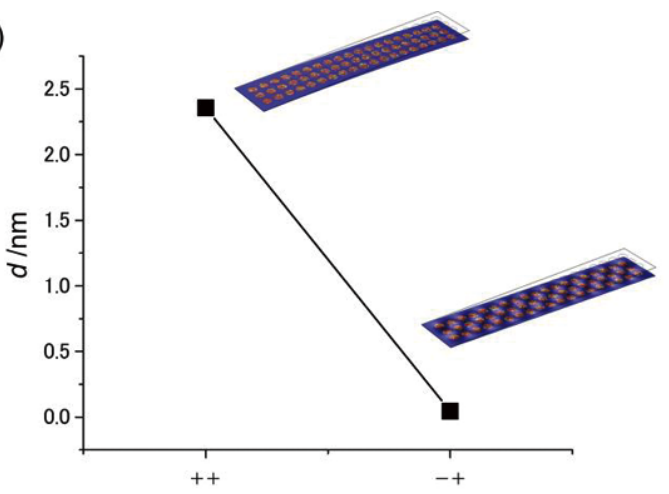

(b)

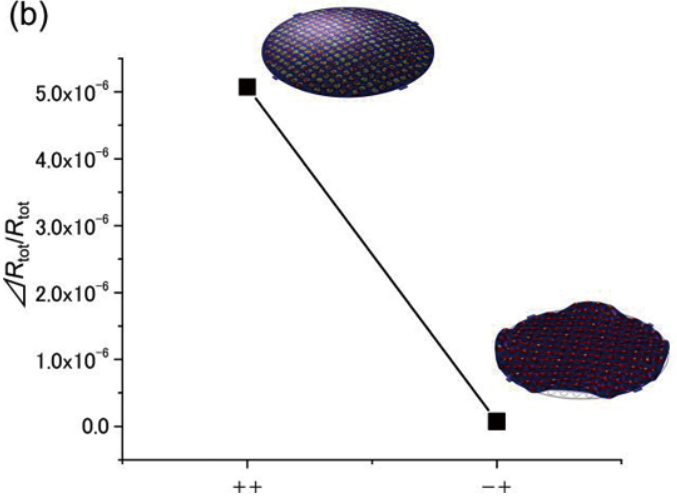

Fig. 8 Dependence of the sensing signals on the phase of cells (in-phase and out-of-phase) for (a) cantilever-type sensor and (b) MSS.

be noted that asymmetric configurations like $x$-aligned cells shown in Figs. 4 and $7 \mathrm{~b}$ lead to non-zero sensing signals even if the cells are out-of-phase. Therefore, the sensing signals depend not only on the phase but also on the configuration.

\section{Discussion}

The presented results indicate that cellular forces associated with cell adhesion can be detected by nanomechanical sensors if cells synchronize their motions. However, it is expected that no practical sensing signals are obtained when cells spread or contract randomly because the surface stress induced by each cell cancels out each other on the sensors. Collective cell motion, in which a group of cells move in concert, is frequently observed in morphogenesis, wound healing, and cancer metastasis, and so on. ${ }^{15-19}$ During such processes, each cell aligns its motion with neighboring cells, leading to the in-phase motions of multiple cells. Therefore, nanomechanical sensors may be utilized to monitor a group of cells in which collective motions are induced. In such a case, the signal amplitude of nanomechanical sensors may be regarded as a degree of collectiveness of cells motion.

Considering that the limits of detection of a cantilever-type sensor and an MSS are $\sim 1 \mathrm{~nm}(=d)$ and $\sim 5 \times 10^{-6}\left(=\Delta R_{\mathrm{tot}} / R_{\mathrm{tot}}\right)$, respectively, ${ }^{7,14}$ it is difficult to obtain clear sensing signals by using these sensors even though cells synchronize their motions (Fig. 8). To enhance the sensing signals, an elastic material coating is known to be effective, ${ }^{20,21}$ We investigated the effect of a coating layer on the sensitivity by FEA simulations. The dependencies on thickness $t$ and Young's modulus $E$ of a coating material in terms of $d$ and $\Delta R_{\mathrm{tot}} / R_{\mathrm{tot}}$ are summarized in Figs. 9 (a)

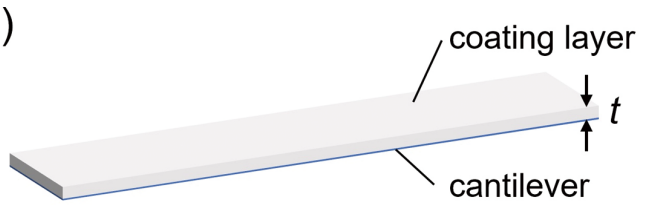

(b)

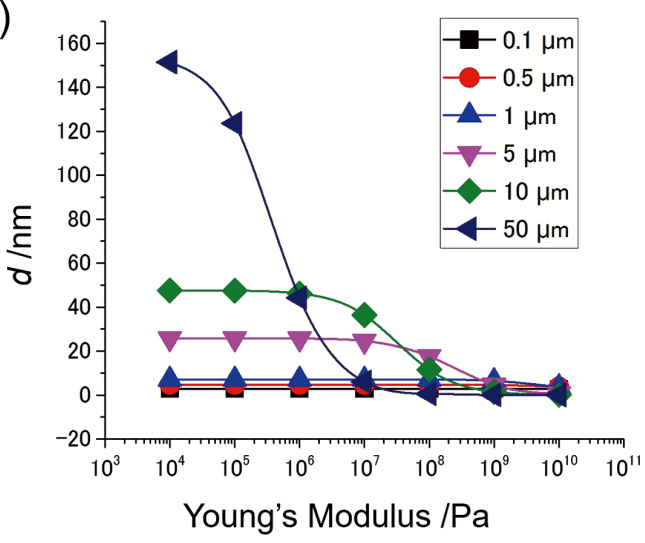

(c)

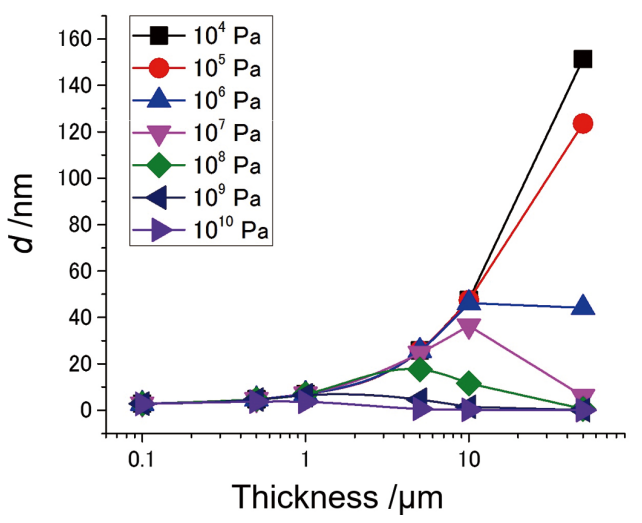

Fig. 9 (a) Schematic illustration of a cantilever-type sensor with a coating layer. Cantilever deflection $d$ as a function of (b) Young's modulus $E$ and (b) thickness $t$.

and 10, respectively. The positive (tensile) surface stress is applied by in-phase multiple cells covering the sensors. It is found that amplified $d$ and $\Delta R_{\mathrm{tot}} / R_{\mathrm{tot}}$ can be achieved by using a softer and thicker coating layer, resulting in sensing signals large enough to be detected. This behavior is quite similar to the case where surface stress is induced on the whole surface of the receptor layer of a cantilever-type sensor. ${ }^{20}$ Note that real cell populations would exhibit non-uniform surface stress on the sensors, giving rise to deviation from the present simulation. It is also worth noting that the sensing signal of an MSS structure is robust against such fluctuation in surface stress compared to a cantilever-type structure. ${ }^{22}$

The results shown in Figs. 9 and 10 are favorable from the view point of analysis of cell motility. Our results imply that commonly-used solid cell culture media can be utilized for coating materials on the nanomechanical sensors; for example, gelatin or collagen. Since the Young's moduli of gelatin and collagen vary ranging from $10^{3}$ to $10^{10} \mathrm{~Pa}$ depending on molecular weight and structure, they are applicable to coatings with high affinity for cells. ${ }^{23-25}$ However, it should be noted that signals may be reduced as a result of stress relaxation for very soft materials since the effect of viscoelastic properties is not taken into account in the present simulation. 
(a)

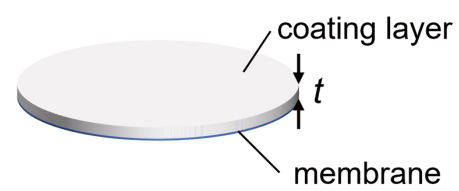

(b)

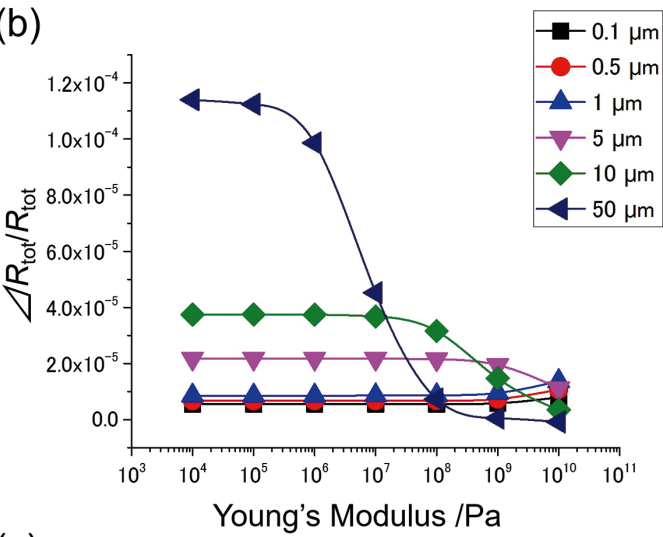

(c)

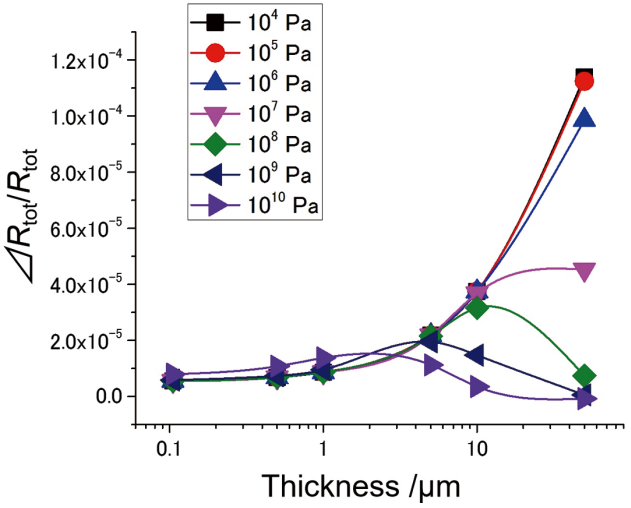

Fig. 10 (a) Schematic illustration of an MSS with a coating layer $\Delta R_{\mathrm{tot}} / R_{\mathrm{tot}}$ as a function of (b) Young's modulus $E$ and (b) thickness $t$

\section{Conclusions}

We performed FEA simulations on the detection of cellular forces by nanomechanical sensors, namely a cantilever-type sensor and an MSS. It becomes clear that cells adhering near the fixed-end lead to larger sensing signals. We also found that the signal intensity depends on the total area covered with cells regardless of the number of cells. For multiple-cell systems, we found that the phase of each cell is important to obtain detectable sensing signals. Cells synchronizing their motions lead to enhanced sensing signals. However the surface stress from each cell cancels out each other when cells spread or contract randomly, resulting in no noticeable sensing signals. We have shown that coatings on the nanomechanical sensors' surface can effectively amplify the sensing signals caused by the cellular forces. The results indicate that typical solid cell culture media can be utilized as a coating material. This study would provide comprehensive information on the applicability of nanomechanical sensors to mechanobiology, especially for the analysis of cell motility.

\section{Acknowledgements}

This research was supported by a Grant-in-Aid for Young
Scientists (B) 16K21602 Ministry of Education, Culture, Sports, Science and Technolgy (MEXT), Japan; a Grant-in-Aid for Scientific Research (B) 15H03588, MEXT, Japan; and the World Premier International Research Center Initiative (WPI) on Materials Nanoarchitectonics (MANA).

\section{References}

1. Z. Long, L. Kou, J. Sepaniak Michael, and X. Hou, Rev. Anal. Chem., 2013, 32, 135.

2. J. L. Arlett, E. B. Myers, and M. L. Roukes, Nat. Nanotechnol., 2011, 6, 203.

3. K. R. Buchapudi, X. Huang, X. Yang, H.-F. Ji, and T. Thundat, Analyst, 2011, 136, 1539.

4. K. Shiba, G. Imamura, and G. Yoshikawa, "Biomaterials Nanoarchitectonics", ed. M. Ebara, 2016, Chap. 4.3, William Andrew Publishing, New York, 177.

5. J. K. Gimzewski, C. Gerber, E. Meyer, and R. R. Schlittler, Chem. Phys. Lett., 1994, 217, 589.

6. T. Thundat, R. J. Warmack, G. Y. Chen, and D. P. Allison, Appl. Phys. Lett., 1994, 64, 2894.

7. G. Yoshikawa, T. Akiyama, S. Gautsch, P. Vettiger, and H. Rohrer, Nano Lett., 2011, 11, 1044.

8. J. H.-C. Wang and B. P. Thampatty, Biomech. Model. Mechanobiol., 2006, 5, 1.

9. J. le Digabel, M. Ghibaudo, L. Trichet, A. Richert, and B. Ladoux, Med. Biol. Eng. Comput., 2010, 48, 965.

10. M. Gupta, L. Kocgozlu, B. R. Sarangi, F. Margadant, M. Ashraf, and B. Ladoux, "Methods in Cell Biology", ed. K. P. Ewa, 2015, Vol. 125, Academic Press, Cambridge, 289.

11. L. E. Dickinson, D. R. Rand, J. Tsao, W. Eberle, and S Gerecht, J. Biomed. Mater. Res. A, 2012, 100, 1457.

12. M.-H. Kim, Y. Sawada, M. Taya, and M. Kino-oka, J. Biol. Eng., 2014, 8, 1 .

13. I. B. Bischofs, S. S. Schmidt, and U. S. Schwarz, Phys. Rev. Lett., 2009, 103, 048101.

14. G. Yoshikawa, T. Akiyama, F. Loizeau, K. Shiba, S Gautsch, T. Nakayama, P. Vettiger, N. Rooij, and M. Aono, Sensors, 2012, 12, 15873.

15. E. Scarpa and R. Mayor, J. Cell Biol., 2016, 212, 143.

16. S. R. K. Vedula, A. Ravasio, C. T. Lim, and B. Ladoux, Physiology, 2013, 28, 370.

17. L. Petitjean, M. Reffay, E. Grasland-Mongrain, M. Poujade, B. Ladoux, A. Buguin, and P. Silberzan, Biophys. J., 2010, 98, 1790.

18. P. Friedl and D. Gilmour, Nat. Rev. Mol. Cell Biol., 2009 , $10,445$.

19. C. J. Weijer, J. Cell Sci., 2009, 122, 3215.

20. G. Yoshikawa, C. J. Y. Lee, and K. Shiba, J. Nanosci. Nanotechnol., 2014, 14, 2908.

21. G. Yoshikawa, Appl. Phys. Lett., 2011, 98, 173502.

22. F. Loizeau, T. Akiyama, S. Gautsch, P. Vettiger, G. Yoshikawa, and N. F. de Rooij, Sens. Actuators, A, 2015, 9 , 228.

23. M. P. E. Wenger, L. Bozec, M. A. Horton, and P. Mesquida, Biophys. J., 2007, 93, 1255.

24. C. E. Schvezov, M. Czerner, L. S. Fellay, M. P. Suárez, P. M. Frontini, and L. A. Fasce, Procedia Mater. Sci., 2015, 8, 287.

25. C. A. Grant, D. J. Brockwell, S. E. Radford, and N. H. Thomson, Biophys. J., 2009, 97, 2985. 